\title{
Lopsided dust rings in transition disks
}

\author{
T. Birnstiel ${ }^{1,2}$, C. P. Dullemond ${ }^{3}$, and P. Pinilla ${ }^{3}$ \\ 1 Excellence Cluster Universe, Technische Universität München, Boltzmannstr. 2, 85748 Garching, Germany \\ 2 Harvard-Smithsonian Center for Astrophysics, 60 Garden Street, Cambridge, MA 02138, USA \\ e-mail: tbirnstiel@cfa.harvard.edu \\ 3 Heidelberg University, Center for Astronomy (ZAH), Institute for Theoretical Astrophysics, Albert Ueberle Str. 2, \\ 69120 Heidelberg, Germany
}

Received 4 December 2012 / Accepted 9 January 2013

\begin{abstract}
Context. Particle trapping in local or global pressure maxima in protoplanetary disks is one of the new paradigms in the theory of the first stages of planet formation. However, finding observational evidence for this effect is not easy. Recent work suggests that the large ring-shaped outer disks observed in transition disk sources may in fact be lopsided and constitute large banana-shaped vortices. Aims. We wish to investigate how effectively dust can accumulate along the azimuthal direction. We also want to find out if the size-sorting resulting from this accumulation can produce detectable signatures at millimeter wavelengths.

Methods. To keep the numerical cost under control we developed a 1+1D method in which the azimuthal variations are treated separately from the radial variations. The azimuthal structure was calculated analytically for a steady-state between mixing and azimuthal drift. We derived equilibration time scales and compared the analytical solutions to time-dependent numerical simulations. Results. We found that weak, but long-lived azimuthal density gradients in the gas can induce very strong azimuthal accumulations of dust. The strength of the accumulations depends on the Péclet number, which describes the relative importance of advection and diffusion. We applied our model to transition disks and our simulated observations show that this effect would be easily observable with the Atacama Large Millimeter/submillimeter Array (ALMA) and could be used to put constraints on the strength of turbulence and the local gas density.
\end{abstract}

Key words. accretion, accretion disks - protoplanetary disks - stars: pre-main sequence - planets and satellites: formation submillimeter: planetary systems - circumstellar matter

\section{Introduction}

The process of planet formation is thought to start with the growth of dust aggregates in a protoplanetary disk through coagulation (see, e.g., the review by Blum \& Wurm 2008). The idea is that these aggregates get successively bigger until either gravoturbulent processes set in (Goldreich \& Ward 1973; Johansen et al. 2007; Cuzzi et al. 2008) or planetesimals are formed directly via coagulation (Weidenschilling 1977; Okuzumi et al. 2012; Windmark et al. 2012). One of the main unsolved problems in these scenarios is excessive radial drift (Weidenschilling 1977; Nakagawa et al. 1986; Brauer et al. 2007). The origin of this problem lies in the sub-Keplerian motion of the gas in the disk, caused by the inward-pointing pressure gradient. The dust particles in the disk, however, feel only the friction with the sub-Keplerian gas and as they grow to larger sizes, the reduced surface-to-mass ratio causes them to fall toward the star with speeds of up to $50 \mathrm{~m} \mathrm{~s}^{-1}$ (Whipple 1972; Weidenschilling 1977). Thus, if a particle grows, it will sooner or later get "flushed" toward the star before it can grow very large (Brauer et al. 2008; Birnstiel et al. 2010, 2012). This is what we call the radial drift barrier, and this still poses one of the main unsolved problems of the early phases of planet formation.

A possible solution to this problem might lie in the concept of "particle traps". This idea was proposed some time ago in the context of anticyclonic vortices by Barge \& Sommeria (1995) and Klahr \& Henning (1997), and in the context of turbulent eddies (Johansen \& Klahr 2005; Cuzzi \& Hogan 2003).
These vortex- or eddy-related local pressure maxima act as particle traps because particles tend to drift towards higher pressure. In the case of vortices and eddies they act on small scales. Particle traps on a global disk scale have been proposed as well (Whipple 1972; Rice et al. 2006; Alexander \& Armitage 2007; Garaud 2007; Kretke \& Lin 2007; Dzyurkevich et al. 2010) and are shown to be conducive to planet formation (Brauer et al. 2008). Intermediate scale particle traps may also occur from magnetorotationally driven turbulence: the so-called zonal flows (Johansen et al. 2009).

If we want to test by observations whether this scenario of particle trapping actually occurs in nature, we are faced with a problem. The terrestrial planet-forming region around a premain sequence star is usually too small on the sky to be spatially sufficiently resolved to test this trapping scenario. Moreover, the optical depth of this inner disk region is likely to be too large to be able to probe the mid-plane region of the disk. Fortunately, what constitutes the "meter-size drift-barrier" at $1 \mathrm{AU}$ is a "centimeter-size drift-barrier" at 50 AU. Those disk regions are optically thin at millimeter $(\mathrm{mm})$ wavelength and particles in the $\mathrm{mm}$ size range can be spectroscopically identified by studying the mm spectral slope (Testi et al. 2001; Natta et al. 2004; Ricci et al. 2010a,b). So the goal that has been pursued recently is to identify observational signatures of dust particle trapping of millimeter-sized particles in the outer regions of disks, as a proxy of what happens in the unobservable inner regions of the disk (Pinilla et al. 2012b,a). The Pinilla et al. (2012a) paper suggests that the huge mm continuum rings observed in most of the 
transition disks (Piétu et al. 2006; Brown et al. 2008; Hughes et al. 2009; Isella et al. 2010; Andrews et al. 2011), may in fact be large global particle traps caused by the pressure bump resulting from, for example, a massive planet opening up a gap.

In these papers we have focused on the intermediate-scale (zonal-flow-type) and the large-scale (global) pressure bumps, simply because current capabilities of $\mathrm{mm}$ observatories (including ALMA) are not yet able to resolve small-scale structures such as vortices. The vortex trapping scenario thus appears to remain observationally out of reach. However, a closer look at the mm maps of transition disks suggest that some of them may exhibit a deviation from axial symmetry. For instance, the observations presented in Mayama et al. (2012) or the mm images of Brown et al. (2009) suggests a lopsided banana-shaped ring instead of a circular ring. Regály et al. (2012) proposed that these banana-shaped rings are in fact a natural consequence of mass piled up at some obstacle in the disk. Once the resulting ring becomes massive enough, it becomes Rossby-unstable and a large banana-shaped vortex is formed that periodically fades and reforms with a maximum azimuthal gas density contrast of a factor of a few. Regály et al. (2012) showed that this naturally leads to lopsided rings seen in mm wavelength maps (see also earlier work by Wolf \& Klahr 2002) on radiative transfer predictions of observability of vortices with ALMA). The formation of such Rossby-wave induced vortices was previously demonstrated by Lyra et al. (2009), who showed that they may in fact (when they are situated much farther inward, in the planet forming region) lead to the rapid formation of planetary embryos of Marsmass. Sándor et al. (2011) subsequently showed that this scenario may rapidly produce a 10 Earth-mass planetary core.

The goal of the current letter is to combine the scenario of forming a lopsided gas ring (e.g., Regály et al. 2012) with the scenario of particle trapping and growth presented by Pinilla et al. (2012a). In Sect. 2 we will outline the physical effects involved and derive analytical solutions to the dust distribution along the non-axisymmetric pressure bump and in Sect. 3 we will test the observability of these structures in resolved (sub-)mm imaging and in the $\mathrm{mm}$ spectral index. Our findings will be summarized in Sect. 4.

\section{Analytical model}

Dust particles embedded in a gaseous disk feel drag forces if they move relatively to the gas. The radial and azimuthal equations of motion have been solved, for example, by Weidenschilling (1977) or Nakagawa et al. (1986) for the case of an axisymmetric, laminar disk. It was found that particles drift inward towards higher pressure. In this paper we will focus on the case where a non-axisymmetric structure has formed a long-lived, non-axisymmetric pressure maximum in the disk. This pressure maximum is able to trap inward-spiralling dust particles. As was done in the aforementioned works, we can solve for a stationary drift velocity, but contrary to the calculations of Nakagawa et al. (1986), the radial pressure gradient is zero at the pressure maximum while the azimuthal pressure gradient can be different from zero. This leads again to a systematic drift motion of the dust particles towards the pressure maximum, but now in azimuthal instead of in radial direction.

The equations of motion in polar coordinates relative to the Keplerian motion become (see Nakagawa et al. 1986)

$$
\begin{aligned}
& \frac{\partial u_{\mathrm{d}, r}}{\partial t}=-A \rho_{\mathrm{g}}\left(u_{\mathrm{d}, r}-u_{\mathrm{g}, r}\right)+2 \Omega_{\mathrm{k}} u_{\mathrm{g}, \phi} \\
& \frac{\partial u_{\mathrm{d}, \phi}}{\partial t}=-A \rho_{\mathrm{g}}\left(u_{\mathrm{d}, \phi}-u_{\mathrm{g}, \phi}\right)-\frac{1}{2} \Omega_{\mathrm{k}} u_{\mathrm{d}, r}
\end{aligned}
$$

$$
\begin{aligned}
& \frac{\partial u_{\mathrm{g}, r}}{\partial t}=-A \rho_{\mathrm{d}}\left(u_{\mathrm{g}, r}-u_{\mathrm{d}, r}\right)+2 \Omega_{\mathrm{k}} u_{\mathrm{g}, \phi} \\
& \frac{\partial u_{\mathrm{g}, \phi}}{\partial t}=-A \rho_{\mathrm{d}}\left(u_{\mathrm{g}, \phi}-u_{\mathrm{d}, \phi}\right)-\frac{1}{2} \Omega_{\mathrm{k}} u_{\mathrm{g}, r}-\frac{1}{r \rho_{\mathrm{g}}} \frac{\partial P}{\partial \phi},
\end{aligned}
$$

where $u_{\mathrm{d}, r}$ and $u_{\mathrm{d}, \phi}$ are the $r$ and $\phi$ components of the dust velocity, respectively, $u_{\mathrm{g}, r}$ and $u_{\mathrm{g}, \phi}$ the $r$ and $\phi$ components of the gas, $\Omega_{\mathrm{k}}$ the Keperian frequency, $P$ the gas pressure, and $\rho_{\mathrm{d}}$ and $\rho_{\mathrm{g}}$ the dust and gas densities; $A$ denotes the drag coefficient (see Nakagawa et al. 1986, Eqs. (2.3) and (2.4)). Solving the above equations for the velocity along the $\phi$ direction at the mid-plane $(z=0)$ gives

$u_{\mathrm{d}, \phi}=\frac{1}{\mathrm{St}+\mathrm{St}^{-1}(1+X)^{2}} \frac{1}{\rho_{\mathrm{g}} V_{\mathrm{k}}} \frac{\partial P}{\partial \phi}$,

where $X=\rho_{\mathrm{d}} / \rho_{\mathrm{g}}$ is the dust-to-gas ratio, $V_{\mathrm{k}}$ the Keplerian velocity, and the Stokes number ${ }^{1}$ is given by

$\mathrm{St}=\frac{\rho_{\mathrm{s}} a}{\rho_{\mathrm{g}} c_{\mathrm{s}} \sqrt{8 / \pi}} \Omega_{\mathrm{k}}$

with $\rho_{\mathrm{s}}$ as internal density of the dust, particle radius $a$, and the isothermal sound speed $c_{\mathrm{s}}$. Dust is advected with the velocity given in Eq. (5), but it is also turbulently stirred. Together, the evolution of the dust density along the ring is then described by

$\frac{\partial \rho_{\mathrm{d}}}{\partial t}=\frac{\partial}{\partial y}\left(\rho_{\mathrm{d}} u_{\mathrm{d}, \phi}\right)-\frac{\partial}{\partial y}\left(D \rho_{\mathrm{g}} \frac{\partial}{\partial y}\left(\frac{\rho_{\mathrm{d}}}{\rho_{\mathrm{g}}}\right)\right)$,

where $y=r \phi$ is the coordinate along the ring circumference. We use a dust diffusion coefficient $D$ according to Youdin \& Lithwick (2007), $D=D_{\text {gas }} /\left(1+\mathrm{St}^{2}\right)$, where we assume the gas diffusivity to be equal to the gas viscosity, taken to be $v=$ $\alpha_{\mathrm{t}} c_{\mathrm{s}}^{2} / \Omega_{\mathrm{k}}$, with $\alpha_{\mathrm{t}}$ as the turbulence parameter (see Shakura \& Sunyaev 1973). Equation (7) can be integrated forward in time numerically, but assuming that the turbulent mixing and the drift term have reached an equilibrium, and also assuming a low dustto-gas ratio, we can analytically solve for the dust density in a steady-state between mixing and drifting, which yields

$\rho_{\mathrm{d}}(y)=C \rho_{\mathrm{g}}(y) \exp \left[-\frac{\operatorname{St}(y)}{\alpha_{\mathrm{t}}}\right]$,

where $C$ is a normalization constant and $\operatorname{St}(y)$ is the Stokes number which depends on $y$ via the changes in gas density. Equation (8) thus predicts the distribution of dust for any given profile of the gas density $\rho_{\mathrm{g}}$. The contrast between the position of the azimuthal pressure maximum and its surrounding then gives

$\frac{\rho_{\mathrm{d}}^{\max }}{\rho_{\mathrm{d}}^{\min }}=\frac{\rho_{\mathrm{g}}^{\max }}{\rho_{\mathrm{g}}^{\min }} \exp \left[\frac{\mathrm{St}^{\min }-\mathrm{St}^{\max }}{\alpha_{\mathrm{t}}}\right]$,

which is plotted in Fig. 1. The Stokes numbers at the pressure maximum and minimum are $\mathrm{St}^{\max }$ and $\mathrm{St}^{\min }$ (note: $\mathrm{St}^{\text {max }}<$ $\left.\mathrm{St}^{\mathrm{min}}\right)$. It can be seen that once the particle's Stokes number becomes larger than the turbulence parameter $\alpha_{\mathrm{t}}$, the dust concentration becomes much stronger than the gas concentration. However, the time scales on which these concentrations are reached can be significant. To get an estimate for this time scale, we compare the advection time scale $t_{\mathrm{adv}}=L / u$ and the diffusion time scale $t_{\text {diff }}=L^{2} / D$ with velocity $u$ and length scale $L$.

\footnotetext{
$1 \mathrm{St}=1$ typically corresponds to particles of $\mathrm{mm}$ to $\mathrm{cm}$ sizes in the outer disk. For typical disk conditions $a \simeq 0.4 \mathrm{~cm} \cdot \mathrm{St} \cdot \Sigma_{\mathrm{g}} / 1 \mathrm{~g} \mathrm{~cm}^{-2}$.
} 


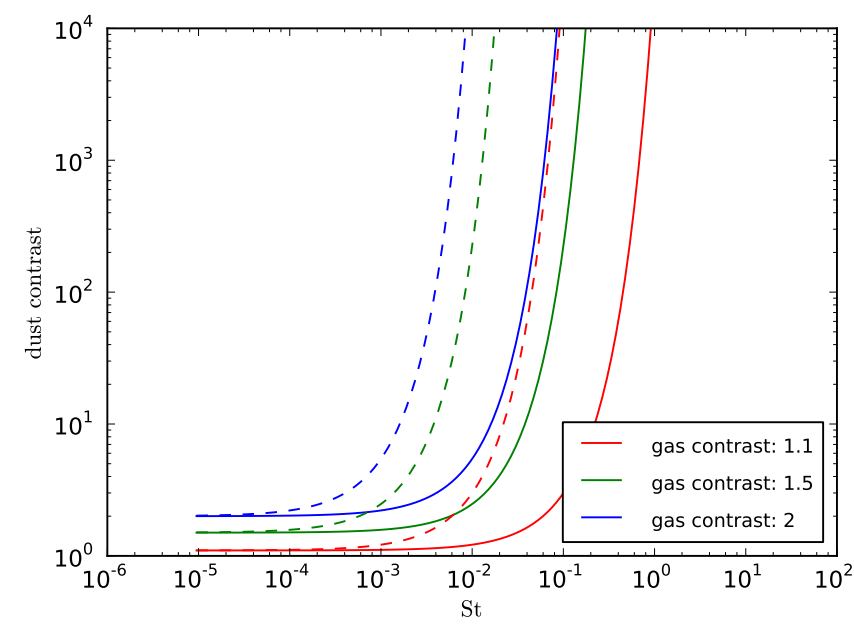

Fig. 1. Contrast between the dust density in the azimuthal maximum and its surroundings for different gas density contrasts $\left(\rho_{\mathrm{g}}^{\max } / \rho_{\mathrm{g}}^{\min }\right)$ and Stokes numbers, as derived in Eq. (9). Turbulence parameters are $\alpha_{\mathrm{t}}=$ $10^{-2}$ (solid) and $\alpha_{\mathrm{t}}=10^{-3}$ (dashed).

The ratio of these time scales is known as the Péclet number Pe and using Eq. (5), can be written as

$\mathrm{Pe}=\frac{t_{\mathrm{diff}}}{t_{\mathrm{adv}}}=\frac{\mathrm{St}}{\alpha_{\mathrm{t}}} \frac{L}{\rho_{\mathrm{g}}} \frac{\partial \rho_{\mathrm{g}}}{\partial y} \simeq \frac{\mathrm{St}}{\alpha_{\mathrm{t}}} \frac{\Delta \rho_{\mathrm{g}}}{\rho_{\mathrm{g}}}$.

It describes the relative importance of advection and diffusion and confirms that dust accumulations occur only for particles with St $\gtrsim \alpha_{\mathrm{t}}$, because otherwise diffusion dominates over advection which means that variations in the dust-to-gas ratio are being smeared out. It also shows that for those large particles the advection time scale is the shorter one, thus setting the time scale of the concentration process, which can be written as

$t_{\mathrm{adv}}=\frac{\pi^{2}}{\delta \mathrm{St}}\left(\frac{H}{r}\right)^{-2} \frac{1}{\Omega_{\mathrm{k}}}$,

where $H=c_{\mathrm{s}} / \Omega_{\mathrm{k}}$ is the pressure scale height, and we have used a mean velocity $\bar{u}=\frac{1}{\pi}\left|\int_{0}^{\pi} u \mathrm{~d} \phi\right|=\frac{c_{\mathrm{s}}^{2}}{\pi V_{\mathrm{k}}} \delta \mathrm{St}$, with

$\delta \mathrm{St}=\left|\operatorname{arccot}\left(\mathrm{St}^{\min }\right)-\operatorname{arccot}\left(\mathrm{St}^{\max }\right)\right|$,

which for $\mathrm{St}<1$ simplifies to $\delta \mathrm{St}=\mathrm{St}^{\min }-\mathrm{St}^{\max }$. Furthermore, we define the pressure maximum and minimum to be at $\phi=0$ and $\phi=\pi$, respectively. As an example, at $35 \mathrm{AU}$, for $H / r=0.07$, a Stokes number of 0.2 , and a gas density contrast of $\rho_{\mathrm{g}}^{\max } / \rho_{\mathrm{g}}^{\min }=2$, the time scale is $3 \times 10^{5}$ years, but could be as short as $10^{2}$ orbits for optimal conditions. Any gas structure therefore has to be long-lived to cause strong asymmetries in the dust, making the asymmetries caused by a planet or longlived vortices the best candidates (Meheut et al. 2012). If such an accumulation is formed and the gas asymmetry disappears, it still takes $t_{\text {diff }}$ to "remove" it, which at $35 \mathrm{AU}$ is of the order of Myrs. It remains to be shown whether short-lived, but reoccurring structures like zonal flows are able to induce strong dust accumulations.

\section{Simulated observations}

In the following, we will evaluate whether the dust structures we expect would be observable in resolved mm images of ALMA.

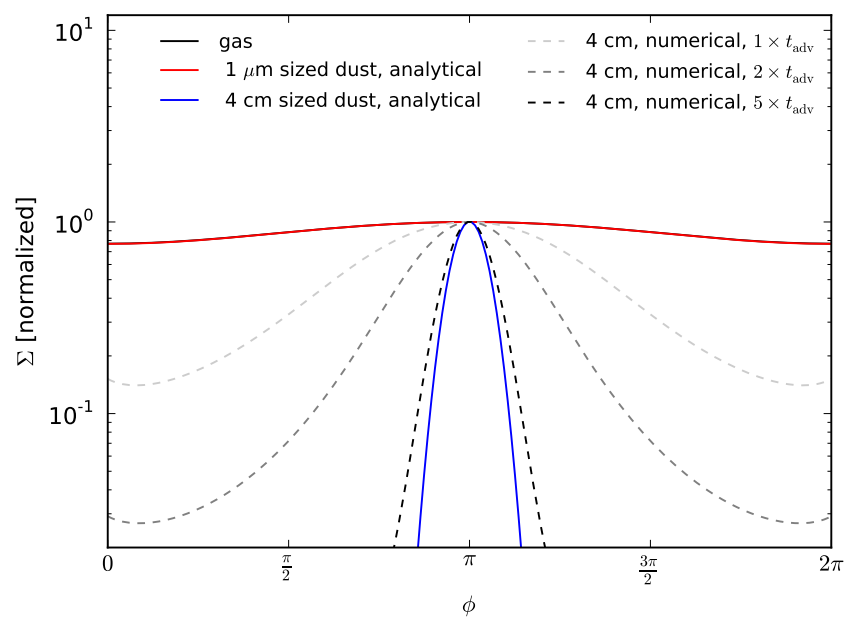

Fig. 2. Azimuthal steady-state solutions for small dust grains (red line) and large dust grains (blue line) for a given sinusoidal gas profile (black line, identical with red line). Dashed lines show numerical solutions at 1,2 , and 5 advection time scales.

We used the results of Pinilla et al. (2012a) for the radial profile of the gas surface density $\bar{\Sigma}_{\mathrm{g}}(r)$ and temperature $T(r)$. These simulations represent a disk of mass $M_{\text {disk }}=0.05 M_{\odot}$ around a solar mass star with a 15 Jupiter-mass planet at 20 AU. For simplicity, as two-dimensional gas surface density we used

$$
\begin{aligned}
& \Sigma_{\mathrm{g}}(r, \phi)=\bar{\Sigma}_{\mathrm{g}}(r)\left[1+A(r) \sin \left(\phi-\frac{\pi}{2}\right)\right] \\
& A(r)=\frac{c-1}{c+1} \exp \left[-\frac{\left(r-R_{\mathrm{s}}\right)^{2}}{2 H^{2}}\right],
\end{aligned}
$$

where $c=\Sigma_{\mathrm{g}, \max } / \Sigma_{\mathrm{g}, \min }$ is the largest contrast of the gas surface density, taken to be 1.5 and $R_{\mathrm{s}}$ is the position of the radial pressure bump. The dust size distribution $\Sigma_{\mathrm{d}}(r, a)$ was also taken from the simulations of Pinilla et al. (2012a) and distributed azimuthally using the analytical solution from Eq. (8) (see Fig. 2). We also confirmed the analytical solution and time scales by solving Eq. (7) numerically, as shown in Fig. 2. Strictly speaking, this analytical solution only holds at the position of the radial pressure bump, but since most of the mm emission comes from the large grains which in the simulations of Pinilla et al. (2012a) are trapped near the radial pressure maximum, this should be a reasonable approximation. Full 2D simulations will be needed to confirm this and to investigate the effects of shear.

To compare directly with current ALMA observations, we calculated the opacities for each grain size at different wavelengths and assumed spherical silicate grains with optical constants for magnesium-iron grains from the Jena database ${ }^{2}$. The continuum intensity maps were calculated assuming that in the sub-mm regime most of the disk mass is concentrated in the optically thin region. We assumed the same stellar parameters as in Pinilla et al. (2012a), azimuthally constant temperature $T(r)$, typical source distances $(d=140 \mathrm{pc})$, and zero disk inclination. We ran ALMA simulations using CASA (v. 3.4.0) at $345 \mathrm{GHz}$ (band 7) and $675 \mathrm{GHz}$ (band 9), shown in Fig. 3. We considered $2 \mathrm{~h}$ of observation, the most extended configuration that is currently available with Cycle 1 , generic values for thermal and atmospheric noises, and a bandwidth of $\Delta v=7.5 \mathrm{GHz}$ for continuum. At these two different frequencies it is possible to detect

\footnotetext{
2 http://www .astro.uni - jena.de/Laboratory/Database/ databases.html
} 


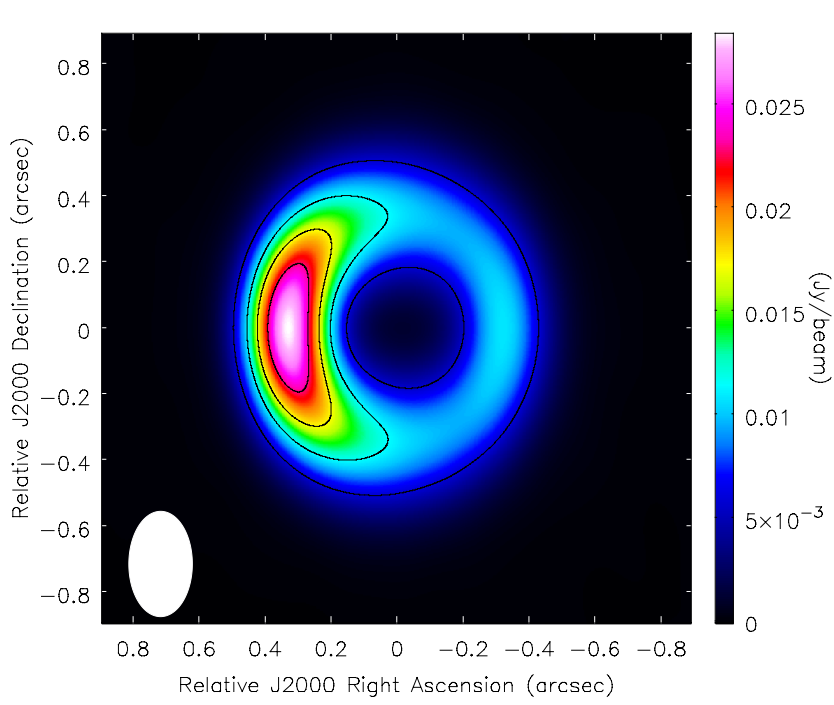

Fig. 3. ALMA simulated images at $345 \mathrm{GHz}$ with an observation time of $2 \mathrm{~h}$. The total flux of the source is $0.13 \mathrm{Jy}$ and the contour lines are at $2,4,6$, and 8 times the rms of $0.22 \mathrm{mJy}$.

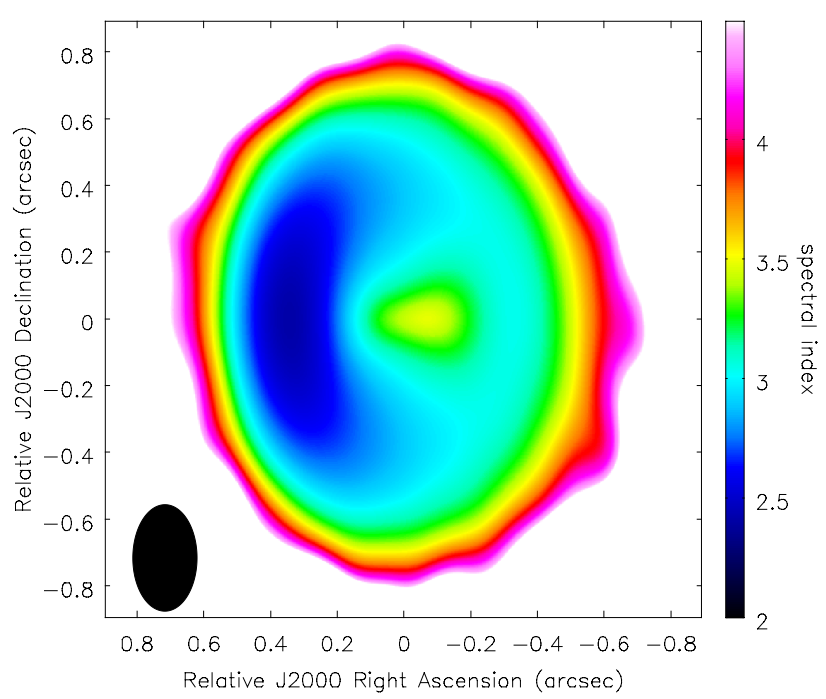

Fig. 4. Spectral index $\alpha_{\mathrm{mm}}$ using simulated images at bands 7 and 9. The antenna configuration was chosen such that the angular resolution is similar for both bands ( 0.16", $22 \mathrm{AU}$ at $140 \mathrm{pc})$.

and resolve regions where the dust is trapped creating a strong azimuthal intensity variation.

The spectral slope $\alpha_{\mathrm{mm}}$ of the spectral energy distribution $F_{v} \propto v^{\alpha}$ is directly related to the dust opacity index at these long wavelengths (e.g., Testi et al. 2003), and it is interpreted in terms of the grain size ( $\alpha_{\mathrm{mm}} \lesssim 3$ implies mm sized grains). With the simulated images in Fig. 3, we compute the $\alpha_{\text {mm }}$ map (Fig. 4), considering an antenna configuration that provides a similar resolution of $\sim 0.16^{\prime \prime}$ ( $\sim 22 \mathrm{AU}$ at $140 \mathrm{pc}$ ) for each band. This resolution is enough to detect $\alpha_{\mathrm{mm}}$ variations along the azimuth, confirming that those are regions where dust accumulates and grows due to the presence of an azimuthal pressure bump.

\section{Summary and conclusions}

We have shown that weak, but long-lived azimuthal asymmetries in the gas density can cause very efficient accumulation of dust at the position of the azimuthal pressure maximum. We have derived analytical steady-state solutions for the dust distribution for any given azimuthal gas density distribution and the time scales on which these distributions develop. Good agreement has been found between the solutions and numerical simulations.

For this dust concentration mechanism to work, particles must grow to sufficiently large sizes $\left(\mathrm{St}>\alpha_{\mathrm{t}}\right.$ ) such that the azimuthal drift becomes stronger than the turbulent diffusion. This typically corresponds to particles of sub-mm to $\mathrm{cm}$ sizes. The strong concentration of the largest grains leads to a sizesorting which is observable via low spectral indices at $\mathrm{mm}$ wavelengths and also in lopsided banana-shaped structures in resolved (sub-)mm images. Finding the size range where the bifurcation between concentration and diffusion happens would put constraints on the turbulence strength and the local gas density of the disk.

Acknowledgements. We thank Ewine van Dishoeck, Simon Bruderer, Nienke van der Marel, Geoffrey Mathews, Hui Li, and the referee for useful comments.

\section{References}

Alexander, R. D., \& Armitage, P. J. 2007, MNRAS, 375, 500

Andrews, S. M., Wilner, D. J., Espaillat, C., et al. 2011, ApJ, 732, 42

Barge, P., \& Sommeria, J. 1995, A\&A, 295, L1

Birnstiel, T., Dullemond, C. P., \& Brauer, F. 2010, A\&A, 513, A79

Birnstiel, T., Klahr, H., \& Ercolano, B. 2012, A\&A, 539, A148

Blum, J., \& Wurm, G. 2008, ARA\&A, 46, 21

Brauer, F., Dullemond, C. P., Johansen, A., et al. 2007, A\&A, 469, 1169

Brauer, F., Henning, T., \& Dullemond, C. P. 2008, A\&A, 487, L1

Brown, J. M., Blake, G. A., Qi, C., Dullemond, C. P., \& Wilner, D. J. 2008, ApJ, 675, L109

Brown, J. M., Blake, G. A., Qi, C., et al. 2009, ApJ, 704, 496

Cuzzi, J. N., \& Hogan, R. C. 2003, Icarus, 164, 127

Cuzzi, J. N., Hogan, R. C., \& Shariff, K. 2008, ApJ, 687, 1432

Dzyurkevich, N., Flock, M., Turner, N. J., Klahr, H., \& Henning, T. 2010, A\&A, 515, A70

Garaud, P. 2007, ApJ, 671, 2091

Goldreich, P., \& Ward, W. R. 1973, ApJ, 183, 1051

Hughes, A. M., Andrews, S. M., Espaillat, C., et al. 2009, ApJ, 698, 131

Isella, A., Natta, A., Wilner, D., Carpenter, J. M., \& Testi, L. 2010, ApJ, 725, 1735

Johansen, A., \& Klahr, H. 2005, ApJ, 634, 1353

Johansen, A., Oishi, J. S., Low, M.-M. M., et al. 2007, Nature, 448, 1022

Johansen, A., Youdin, A., \& Klahr, H. 2009, ApJ, 697, 1269

Klahr, H. H., \& Henning, T. 1997, Icarus, 128, 213

Kretke, K. A., \& Lin, D. N. C. 2007, ApJ, 664, L55

Lyra, W., Johansen, A., Klahr, H., \& Piskunov, N. 2009, A\&A, 493, 1125

Mayama, S., Hashimoto, J., Muto, T., et al. 2012, ApJ, 760, L26

Meheut, H., Keppens, R., Casse, F., \& Benz, W. 2012, A\&A, 542, A9

Nakagawa, Y., Sekiya, M., \& Hayashi, C. 1986, Icarus, 67, 375

Natta, A., Testi, L., Neri, R., Shepherd, D. S., \& Wilner, D. J. 2004, A\&A, 416, 179

Okuzumi, S., Tanaka, H., Kobayashi, H., \& Wada, K. 2012, ApJ, 752, 106

Piétu, V., Dutrey, A., Guilloteau, S., Chapillon, E., \& Pety, J. 2006, A\&A, 460, L43

Pinilla, P., Benisty, M., \& Birnstiel, T. 2012a, A\&A, 545, A81

Pinilla, P., Birnstiel, T., Ricci, L., et al. 2012b, A\&A, 538, A114

Regály, Z., Juhász, A., Sándor, Z., \& Dullemond, C. P. 2012, MNRAS, 419, 1701

Ricci, L., Testi, L., Natta, A., \& Brooks, K. J. 2010a, A\&A, 521, A66

Ricci, L., Testi, L., Natta, A., et al. 2010b, A\&A, 512, A15

Rice, W. K. M., Armitage, P. J., Wood, K., \& Lodato, G. 2006, MNRAS, 373, 1619

Sándor, Z., Lyra, W., \& Dullemond, C. P. 2011, ApJ, 728, L9

Shakura, N. I., \& Sunyaev, R. A. 1973, A\&A, 24, 337

Testi, L., Natta, A., Shepherd, D. S., \& Wilner, D. J. 2001, ApJ, 554, 1087

Testi, L., Natta, A., Shepherd, D. S., \& Wilner, D. J. 2003, A\&A, 403, 323

Weidenschilling, S. J. 1977, MNRAS, 180, 57

Whipple, F. L. 1972, From Plasma to Planet, 211

Windmark, F., Birnstiel, T., Ormel, C. W., \& Dullemond, C. P. 2012, A\&A, 544, L16

Wolf, S., \& Klahr, H. 2002, ApJ, 578, L79

Youdin, A. N., \& Lithwick, Y. 2007, Icarus, 192, 588 